\title{
LIGHT DEVIATION BASED OPTICAL TECHNIQUES APPLIED TO SOLID PROPELLANT COMBUSTION
}

\author{
F. Cauty ${ }^{1}$, C. Eradès ${ }^{1}$, and J.-M. Desse ${ }^{2}$ \\ ${ }^{1}$ Office National d'Études et de Recherches Aérospatiales \\ DEFA/PRS Palaiseau, France \\ ${ }^{2}$ Office National d'Études et de Recherches Aérospatiales \\ DAAP/MMHD Lille, France
}

\begin{abstract}
The Investigation in Combustion of Energetic Materials (InCoME) program is aimed at validating the numerical simulation of composite propellant combustion using nonintrusive optical techniques. The Focusing Schlieren Technique (FST) was selected; it allows catching light deviation from a thin vertical planar section centered above the propellant combustion surface. The optical system is described in the paper. Significant results are presented showing the capabilities of this technique when applied to solid propellant combustion in terms of studying flame structure, flame propagation, and particle tracking.
\end{abstract}

\section{INTRODUCTION}

In the framework of solid-propellant combustion diagnostics, most of the classical experimental techniques are devoted to studies of the solid phase; namely, burning rate with, i. e., ultrasound technique [1], pressure measurement coupled to indirect or direct techniques and/or analysis methods (unsteady behavior) [2]. Studied here is the combustion of solid composite propellants which are a mixture of several ingredients such as ammonium perchlorate (AP), hydroxyl-terminated polybutadiene (HTPB) binder, and metallic loads like Aluminum particles. The InCoME program was aimed at validating the numerical simulation of the composite propellant combustion using nonintrusive optical techniques. Three investigation axes were defined: surface heterogeneity and morphology, species concentration and temperature profile or field, and flame structure $[3,4]$.

This paper is focused on the work done in the frame of the third axis related to the flame/gas structure above the solid propellant surface in regression. Light

This is an Open Access article distributed under the terms of the Creative Commons Attribution-Noncommercial License 3.0, which permits unrestricted use, distribution, and reproduction in any noncommercial medium, provided the original work is properly cited. 
deviation optical techniques were selected: shadowgraph, Schlieren, and interferometric. Each of them is based on the light deviation due to the variation of the refractive index gradients along the optical path. A preliminary work showed the capabilities of these techniques when applied to combustion process studies: the light emitted by the combustion does not affect the image contrary to classical visualization methods, where the high-speed videocamera has to be adjusted with respect to the most luminous part or portion of the scene thus hiding some information. The size of the ingredients of any solid composite propellant is quite small: from a few micrometers for the fine AP or/and additive charges to a few hundreds of micrometers for coarse AP grains. A diffusion flame is associated to each AP grain; this conical shape flame is due to the combustion of the binder and AP decomposition gases. The field of view is relatively small and the spatial resolution is an important parameter. The combustion of a composite solid propellant is unsteady in time and space. The AP grains are always moving and changing their size at the burning surface. This requires an experimental tool having a high-level time resolution. Below, the tested optical techniques are described and commented; a series of examples are presented highlighting the most adapted technique, namely, the Focusing (or Converging) light Schlieren Technique which allows catching light deviation from a thin planar section centered above the propellant sample.

\section{LIGHT DEVIATION BASED OPTICAL SYSTEMS}

\subsection{Shadowgraphy and Differential Interferometry Techniques}

There are a lot of different optical techniques behind these optical generic names [5]. First, the classical shadowgraph or Schlieren technique was tested. All are based on the light deviation induced by the variations in the refractive index $(n)$ along the optical path $(x)$. The refractive index is linked to the density of the gases via Gladstone-Dale coefficients: shadowgraphy corresponds to $d^{2} \rho / d x^{2}$ and Schlieren techniques to $d \rho / d x$.

The CHAMADE named experimental test setup allows igniting pieces of solid propellant using a $\mathrm{CO}_{2}$ laser beam inside a pressurized chamber equipped with two large windows. The light emitted by a white light source enters on one side and the deviated rays are collected at the opposite side of the chamber.

The shadowgraphy system was a simple crossing of the test section using two Clairaut lenses (Fig. 1). The shadowgraphy was performed using two different configurations: one with a continuous white light source $(150 \mathrm{~W})$, the other with a laser pulsed source. Images were collected using a high-speed videocamera.

Two Wollaston's prisms were added moving the system to a differential interferometry (writings in frames in Fig. 1). The color scale of the image was proportional to the optical path difference. More details are given in [6]. 


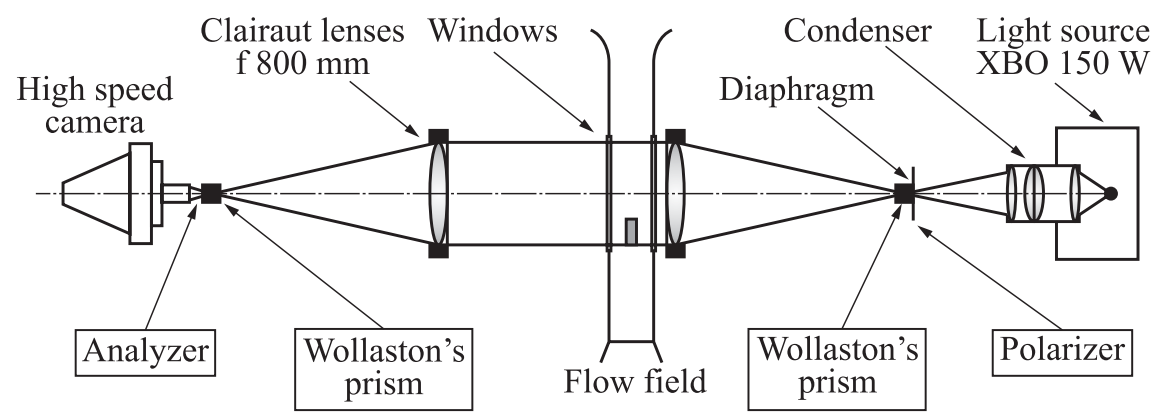

Figure 1 Schematic of shadowgraph and differential interferometry system

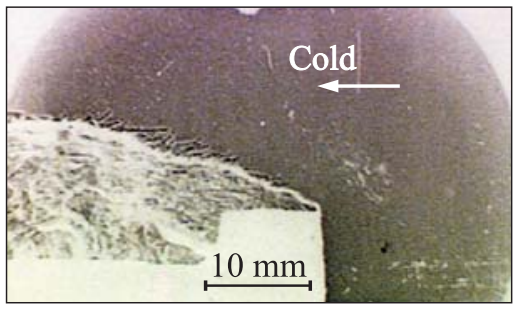

(a)

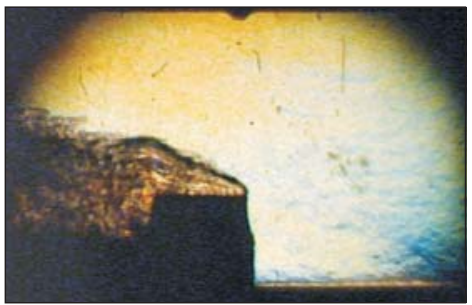

(b)

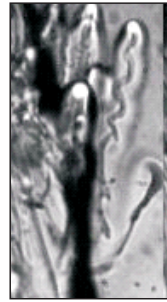

(c)

Figure 2 Shadowgraph (a), differential interferometry (b) images, and shadowgraph zoom on $\mathrm{Al}$ particles (c). (Refer Cauty et al., p. 123.)

The propellant sample was mounted at the center of the bottom wall of the test chamber. The propellant sample was a "sugar-like" stick $(5 \times 30$ $\times 5 \mathrm{~mm}$ ). The last dimension is that corresponding to the optical path direction. Samples were exposed to the upstream cold-air flow. Examples of images are shown in Fig. 2 for a smaller $\mathrm{AP} / \mathrm{HTPB} / \mathrm{Al}$ propellant stick put over another propellant stick without Aluminium particles. Despite the boundary between hot and cold gases was easily determined, it was not easy to examine in details the mixing and particle behavior inside hot gases. Aluminum particles were seen injected in the cold flow. Using high-speed camera, it was possible to describe the oscillation of the hot gas boundary linked to the head angle of the sample. The particle trace analysis was also very interesting because the trajectory of a particle was printed by thermal exchange during its motion. The spinning of particles due to the Alumina cap was also seen. The optical path was too long and, since the propellants of industrial type were burned, there were too many particles and flames mixing up all together in the image. The field of view was too large and the spatial resolution was not adapted. These first experimental campaigns provided some information about the advantages of these optical light 
deviation techniques when applied to combustion of energetic materials. Qualitative images integrating the light deviation all along the optical path through the chamber were obtained. The main issues in this technique were too many particles and too much three-dimensional (3D) heat transfer signature in the field. Quantitative analysis was not attainable.

\section{$2.2 \quad$ Focusing Schlieren Technique}

Aiming at reducing the depth of focus, thus the integrated path, an FST system was defined and mounted around the test rig. The principle of this variation of the Schlieren technique is described in details in Weinstein's papers $[7,8]$. An extended light source is required and the Fresnel field lens has to focus the light source at the image lens location. The source grid is composed of knifeedge parallel stripes; their number has to be adjusted, avoiding diffraction effect (too many stripes) and giving enough multiple light sources. The cutoff grid is designed as the negative image of the source grid (Fig. 3).

The vertical displacement of this grid at the location of the source grid image allows adjusting the light deviation limit angle and light and contrast levels. The light emitted by the combustion process is cut by adjusting the position of the cutoff grid (light threshold level). This position depends upon the light (or temperature) level of the combustion (i.e., aluminum particle burning, alumina cap, etc.). There is one focus position for each flow field distance which will cause the flow features to be at a single common location in the image plane. By moving the image plane back and forth, the investigated flow field plan is changed. Depending upon the position of the parallel stripes, the optical index gradient orientation is modified: vertical stripes mean horizontal light deviation gradients. The FST system aimed at obtaining images of the vertical gradients is shown in Fig. 4; the grids have 5 slots. The objective is to obtain a depth of focus smaller than the width of the sample in the light direction (see Fig. 4). As

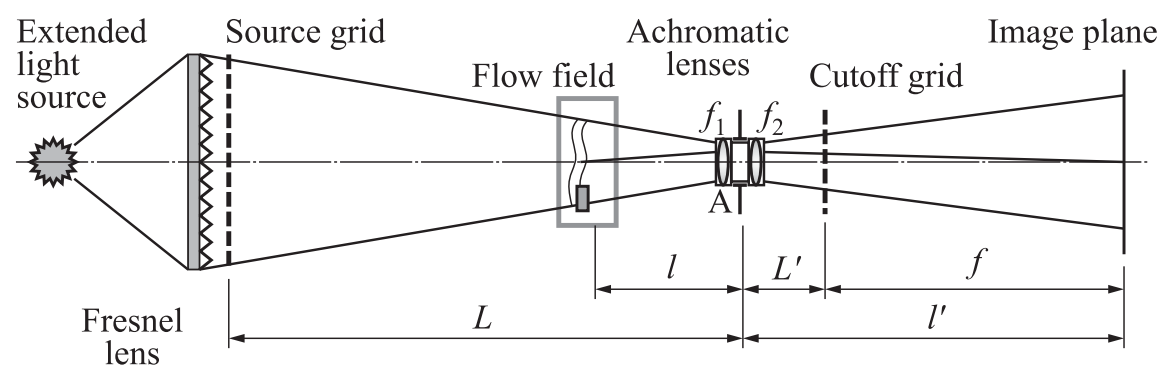

Figure 3 Schematic of the FST optical system 

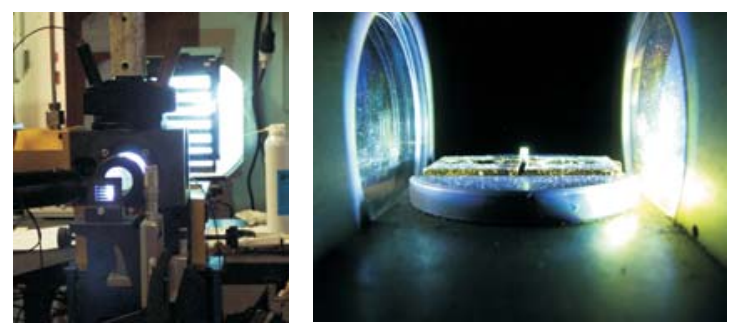

Figure 4 View of the optical system from the camera side and propellant sample at the measurement location. (Refer Cauty et al., p. 125.)

the light deviation is only integrated in a short distance, analyzing the images should be easier than using a classical Schlieren technique.

\section{APPLICATIONS TO SOLID PROPELLANT COMBUSTION}

\subsection{Flames of Ammonium Perchlorate Grain}

The aim of the first FST campaign was to gain experience in this technique. It was decided to test monomodal AP propellant with a maximum AP load. Two coarse AP sizes (200 and $400 \mu \mathrm{m})$ were selected. The main issue was the good reproducibility in the grain combustion sequences.

The selected optical fitting gave a depth-of-focus of $0.46 \mathrm{~mm}$ and a spatial resolution of $70 \mu \mathrm{m}$ which is less than the diameter of the selected AP grains $(200$ or $400 \mu \mathrm{m})$ and a field of view small enough compared to the size of the flames. In Figs. 5 and 6, the videoimages have a width of $0.7 \mathrm{~mm}$ and a height of $1.4 \mathrm{~mm}(256 \times 512$ pixels $)$; they are obtained using a Photron ${ }^{\circledR}$ high-speed videocamera (8.000 frames per second and exposure time 1/140,000 s). The samples of propellant are very thin $(3(400 \mu \mathrm{m})$ AP network layers $)$ but thick enough compared to the depth-of-focus.

The vertical grids, giving horizontal index gradients, underlined more triangular structures which could be understood as due to the diffusion flame. The question was which limits were exhibited by the gradients: the internal zone before the combustion of the first-step dissociated chemical species or the external limit at the end of species reactions. When the field of view was focused at the burning surface level, the images of the HTPB degradation and AP grain combustion could be obtained in tiny details. Figure 7 shows an example of the combustion surface time evolution of a $(400 \mu \mathrm{m})$ AP/HTPB propellant thin slice. Less than $3 \mathrm{AP}$ grains were fixed in the field which was the same as that 


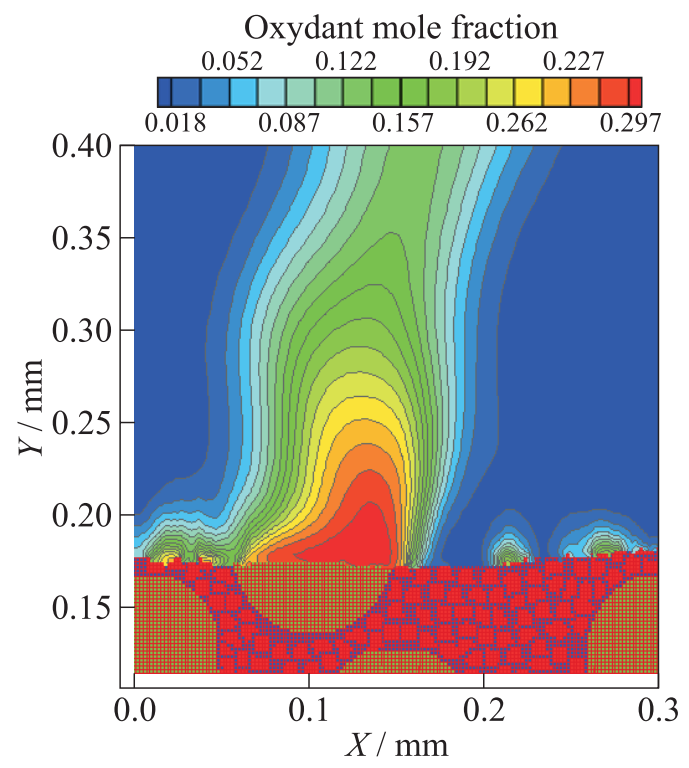

Figure 5 Ammonium perchlorate grain combustion flame: simulation (AP sizes 9 and $90 \mu \mathrm{m})$. (Refer Cauty et al., p. 126.)
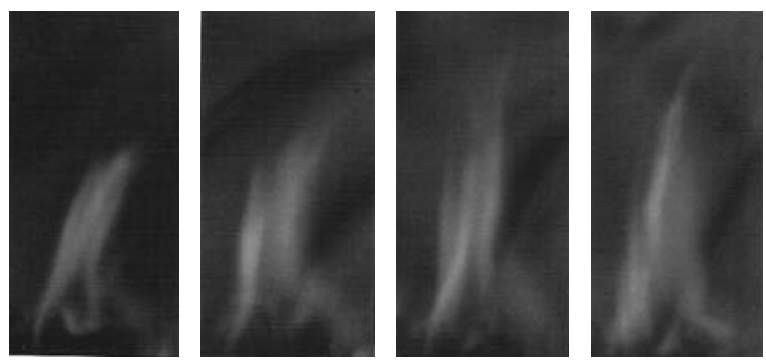

Figure 6 Ammonium perchlorate grain combustion flame: FST sequence $(P$ $=1.5 \mathrm{MPa} ; d t=2.5 \mathrm{~ms}$; and field of view $=0.7 \times 1.4 \mathrm{~mm}$ )

images in Fig. 6. Small AP grain residue was lifting up by the combustion gases and HTPB filaments were seen like a spider on the top of a large AP grain. In that case, the frame rate was 12,500 frames per second and the exposure time was $1 / 100,000 \mathrm{~s}$. The grid stripes were mounted horizontally aiming at giving the image from vertical index gradients.

With a coarse AP grain, conical diffusion flames were observed which were cut by the depth of focus plane. The image does not stand exactly at the sym- 


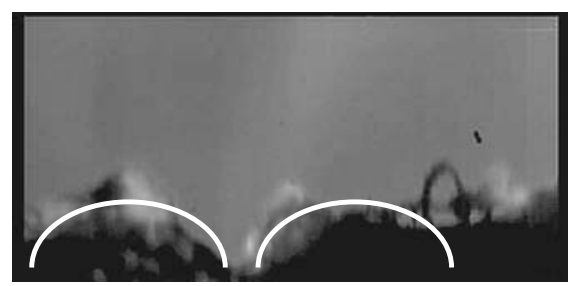

(a)

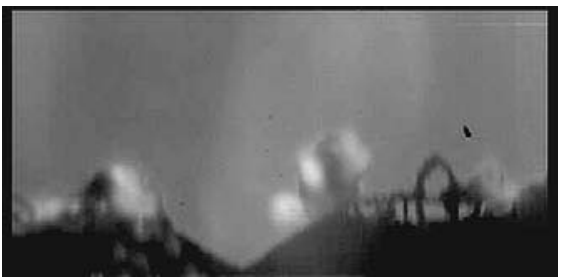

(c)

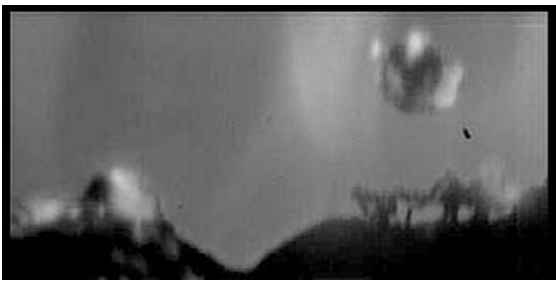

(e)

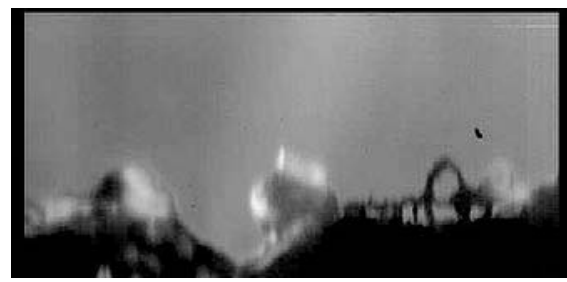

(b)

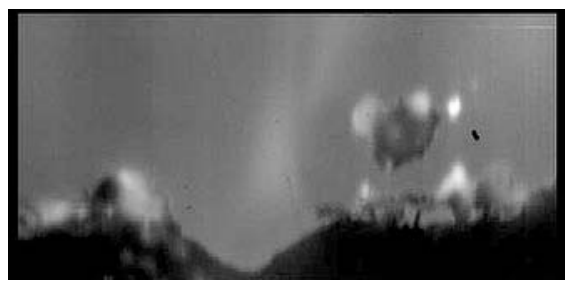

$(d)$

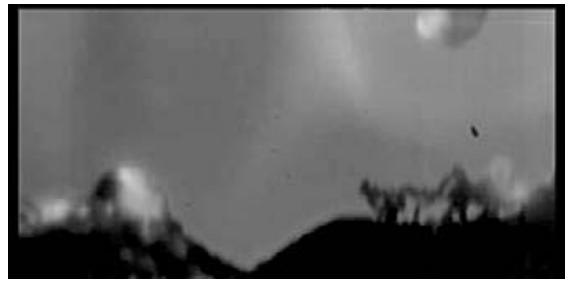

$(f)$

Figure 7 Burning surface of HTPB/AP propellant by FST $(\delta t=0.8 \mathrm{~ms})$

metry axis of each AP grain flame. Using prilled AP sticks and HTPB layers, sandwiches were prepared. Each layer had a thickness of 0.5 to $0.7 \mathrm{~mm}$. The FST images of such sandwiches during their burning showed a "W" structure anchored, as expected, at the interfaces between HTPB and AP. Figure 8 shows images at two pressure levels. The contrast was deeper at $1.5 \mathrm{MPa}$ indicating stronger gradients. The analysis of such images requires the knowledge of the optical refractive indexes of the gases at their temperature. The goal is to couple the FST analysis to the numerically simulated

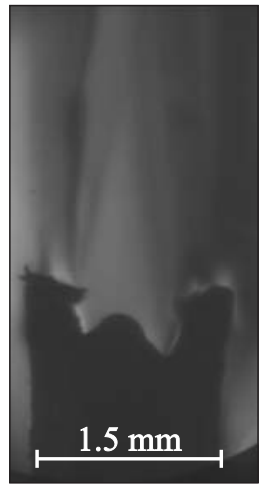

(a)

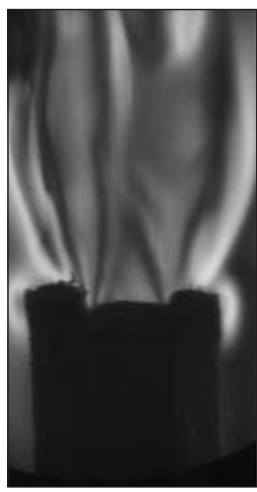

(b)
Figure 8 The HTPB/AP/HTPB sandwiches at $0.5(a)$ and $1.5 \mathrm{MPa}(b)$ 
fields of physical quantities (species concentration, temperature, etc.); this is under investigation [9].

Some clues indicating that the light was deviated by the variation of density of the binder itself in a nonaluminized composite propellant mixture were also got. This implied that FST could be used to investigate the degradation process and the regression in the solid phase near the burning surface. The issue is related to the flatness of the burning surface and the mixing which could occur between the degradation propagation and the orientation of the burning surface [9].

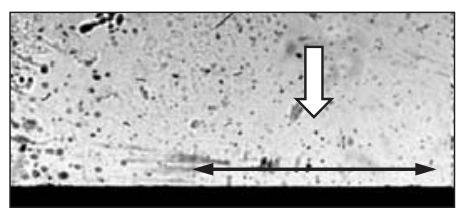

(a)

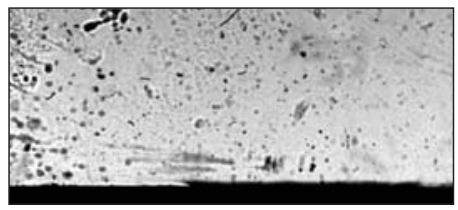

(c)

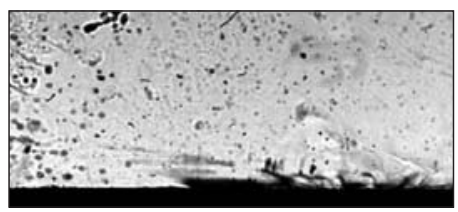

(e)

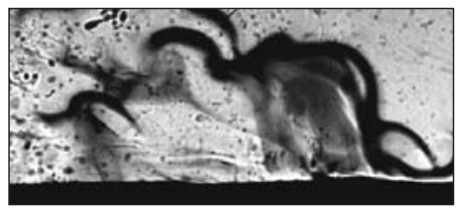

$(g)$

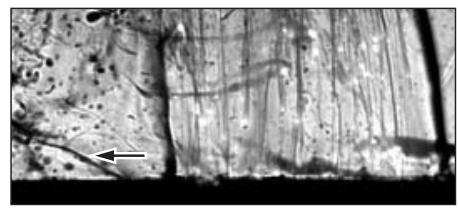

(i)

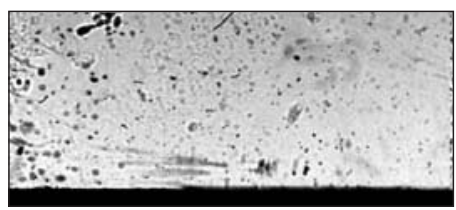

(b)

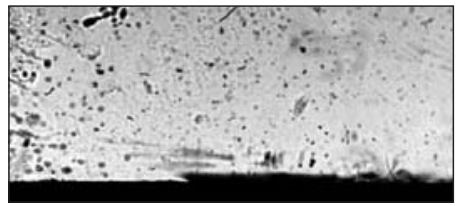

(d)

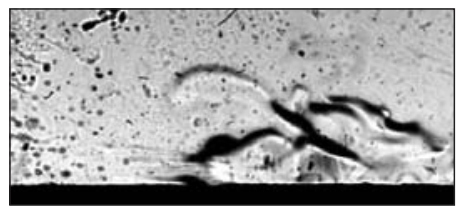

$(f)$

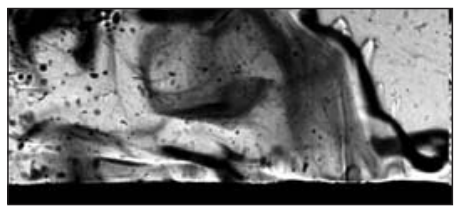

(h)

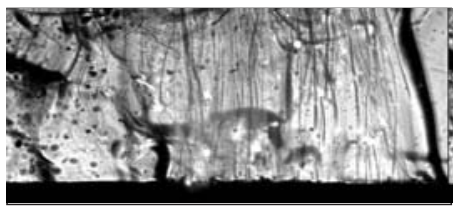

(j)

Figure $9 \mathrm{HTPB} / \mathrm{AP} / \mathrm{Al}$ propellant ignition phase and flame propagation by shadowgraphy 


\subsection{Ignition and Flame Propagation}

The main advantage of these optical techniques is that the image is (quasi) not affected by the combustion emitted light itself. This led to noticeable results concerning ignition and flame propagation phases. In Fig. 9, the classical shadowgraphy images sequence is read line by line from left to right. This is the ignition phase of an aluminized propellant ignited by a $\mathrm{CO}_{2}$ laser beam. The view field is $10 \times 5 \mathrm{~mm}(512 \times 256$ pixels $)$ and the time interval between two images is $1 \mathrm{~ms}$. From the beginning of the beam impact, a back line is rising up from the surface (images $(a)$ to $(d)$ ) due to laminar convection process as a "mirage in the desert" does.

The height of this convective heating increases, and the first convective bubbles appear. They grow up (image $(e)$ to $(g))$ and the ignition starts somewhere between these images. In image $(h)$, the first aluminum particles are seen ejected far away from the surface. The ignition is located at the surface exposed to $\mathrm{CO}_{2}$ laser, the combustion propagates at the burning rate level speed: there is only a low convective heat transfer due to the purge (towards the left hand side) and the combustion gases generated by the ignited zone. The roughness of the surface changes showing the effect of the burning surface heterogeneity underlined by a fine white line.

\subsection{Particle Tracking}

The FST was also used for investigating the behavior of "industrial" propellants at the burning surface level and above. This was the case for composite propellant including nano-Al particles, for composite propellant with different AP grain size mixing aiming at validating experimentally the well-known "pocket model" (particle agglomeration process). The nano-Al propellant had been studied only using Schlieren technique $(256 \times 256$ pixels $)$. The light deviation occurred all along the optical path in the chamber. It showed numerous out-of-focus particles flowing in the chamber (Fig. 10). Despite the quality of the images in Fig. 10 is quite poor, they allow the agglomeration process of nano-Al particles to be understood as creating "flakes" at the burning surface level. This seems to be linked to the batching process.

The study was focused on the Aluminum agglomeration process at the surface and/or the shape of the ejected particles and their tracking (size, displacement, and velocity). Two examples are presented in this paper. The first one is related to the combustion of a composite propellant $[(60+6) \%$ of $\mathrm{AP}, 16 \%$ of HTPB, and $18 \%$ of $\mathrm{Al}(30 \mu \mathrm{m})]$ at $0.5 \mathrm{MPa}$. Ninety percent of AP had the same grain size repartition. With respect to the "pocket model," no agglomeration was expected. This propellant is called "type 1." The FST images are shown in Fig. 11 (top). The field of view is $2.72 \times 1.36 \mathrm{~mm}(1024 \times 512$ pixels $)$ which 


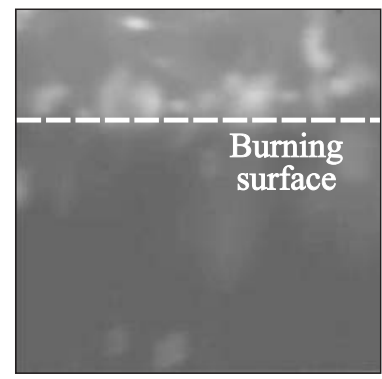

(a)

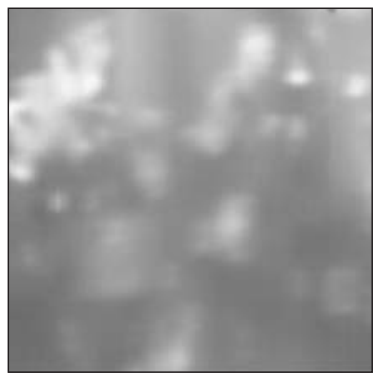

(b)

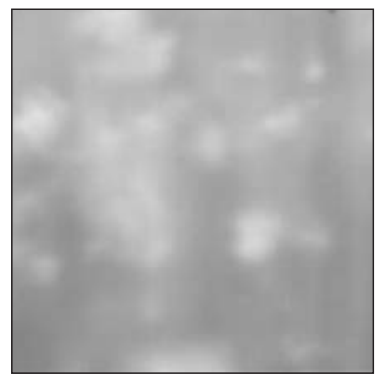

(c)

Figure 10 Composite propellant with nano-Al by Schlieren technique

corresponds to a spatial resolution of $2.65 \mu \mathrm{m} /$ pixel. The time between two images is $0.2 \mathrm{~ms}$ (frame rate $=5,000$ ).

The $\mathrm{Al}$ particles seem to be small in this image; their alumina plumes are very long. Nonburning agglomerates are seen hang to the surface or just ejected. The coloured lines are from the particle tracking process based on ImageJ free code supported by the National Institute of Health (USA) [10]. The trajectories of selected particles were computed, frame by frame. Except for few (compared to the numerous particles ejected) agglomerates, the mean particle size was roughly close to $30 \mu \mathrm{m}$.

The particles can be extracted from the "noise" of the images by subtraction of one image before ignition (Fig. 11, bottom). The burning rate is determined from the levels of the burning surface in the proceeded image. The particle size becomes more precise but the pixel resolution indicates that one particle could be described only by 10 pixels. There is a zoom done on one of these particles in Fig. 12. The selected time and spatial resolutions are not far from the limits for this kind of propellant. The displacement rate of the particles is high: a particle is seen only in few successive images. Thus, the frame rate would have been higher. Note that the field of view could not be enlarged due to the spatial resolution.

Figure 12 shows FST images for the Ariane 5 composite propellant (multimodal AP and $18 \% \mathrm{Al}, 30 \mu \mathrm{m})$. It is well known that this propellant, called "type 2" herein, is very sensitive to agglomeration. This was checked using the FST images. The field of view in these studies was larger $(1 \times 8 \mathrm{~mm}$ $128 \times 1024$ pixels). The frame rate was increased to 15,000 frames per second leading to a time between two images equal to $0.067 \mathrm{~ms}$, as shown in Fig. 12. The pressure was $0.5 \mathrm{MPa}$, i. e., the same as for the "type 1" propellant. Only a few particles are seen in the field but the time and spatial resolutions provide some information about the size of the particle core, its surrounding heat exchange sphere, the alumina cap, its rotation, and the dimension of the plume. 

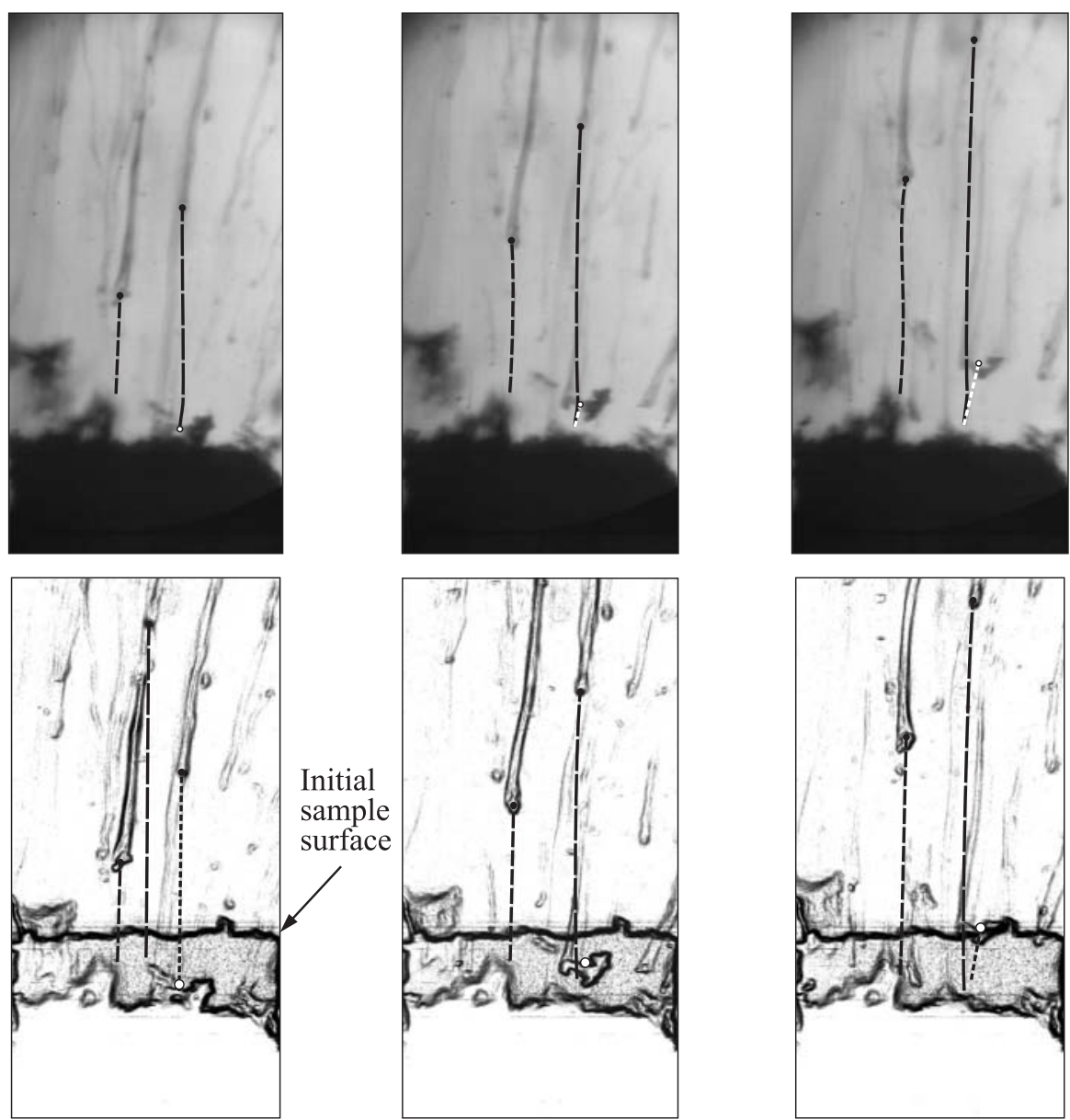

Figure 11 Particle tracking for the "type 1" propellant without and with FST image processing

Tracking selected particles gave their displacement, velocity, and diameter variation. The velocity of "type 2" propellant particles and their diameter are plotted in Fig. 13 as a function of the distance to the surface. This is the first result corresponding to the image processing code which has to be improved and validated (in terms of detection and tracking error and uncertainty). As a result, the capability of FST to track particles even for Aluminum load up to $18 \%$ as that of Ariane 5 booster propellant was validated. The optical path is not an issue anymore. One pixel corresponds to 2.5 to $10 \mu \mathrm{m}$ or more depending upon the selected field. The dimensions of the field of view and the frame rate 

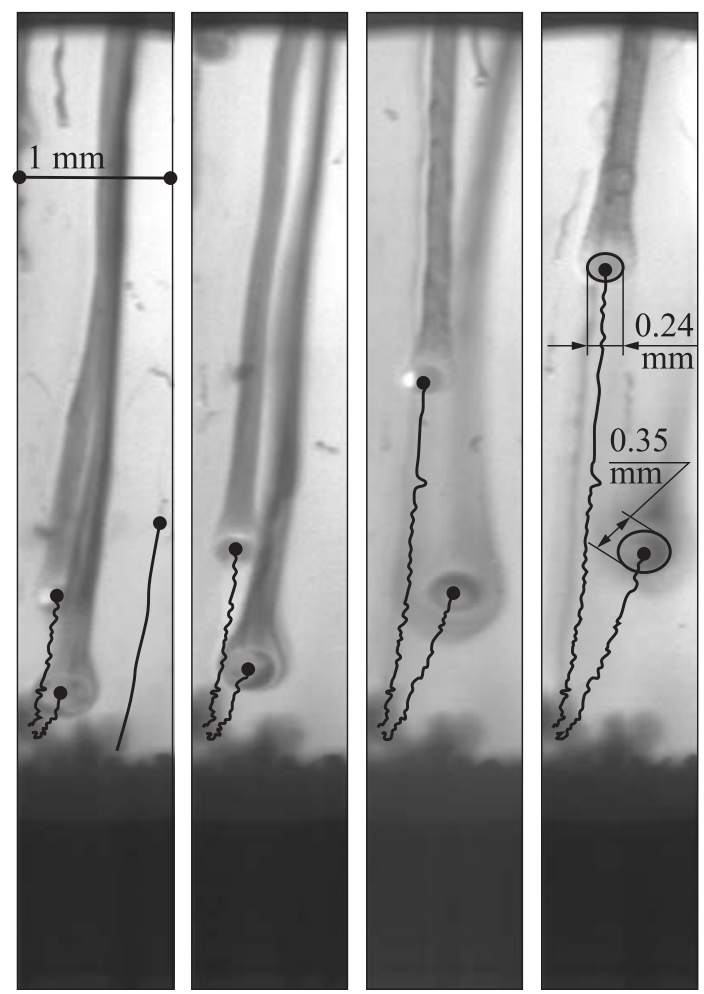

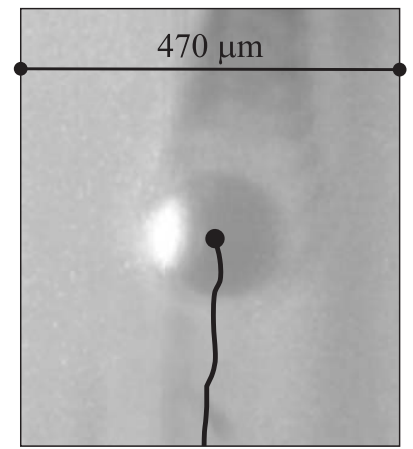

Type 2

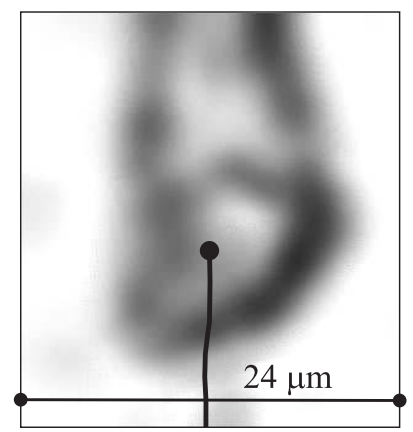

Type 1 (see Fig. 11)

Figure 12 Particle tracking for the "type 2" and single aluminum particle ("type 1" and "type 2" propellants)

of the camera have to be adapted to the expected size of particles. There is a compromise to be found between the size of agglomerates and particles and the level of burning rate at a given pressure.

\section{CONCLUDING REMARKS}

Thus, the FST based on the first derivative function of the optical indexes (gas density) with short-distance light-deviation integration provided very promising results summarized below:

- the quantitative analysis requires a FST simulation routine coupled to the results of solid-propellant combustion numerical simulation;

- the qualitative analysis is worth to be underlined; 

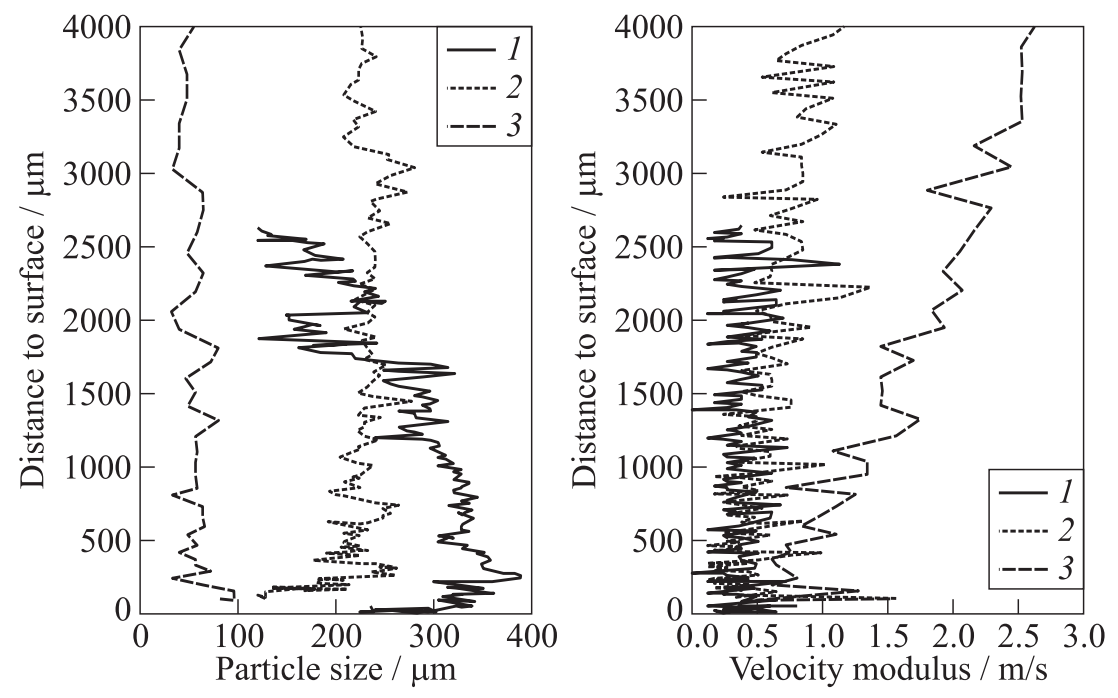

Figure 13 Results of particle tracking for "type 2" propellant: $1-240 \mu \mathrm{m} ; 2$ $150 ;$ and $3-80 \mu \mathrm{m}$

- the light emitted by the combustion process does not affect the FST images (cutoff grid adjustment);

- the heat exchange above the propellant sample is seen in the ignition process; and

- the particles are tracked in the combustion gases; it is not depending upon the particle ratio in the image as for classical visualization.

This technique has to be promoted as an investigation tool in the domain of solid propellant combustion.

\section{ACKNOWLEDGMENTS}

All the experiments were performed in the framework of the InCoME program which was financially supported by ONERA.

\section{REFERENCES}

1. Cauty, F. 2004. The ultrasound waves: A measurement tool for energetic material characterization. AIAA Paper No. 2004-4057. Fort Lauderdale, USA. 
2. Cauty, F. 1999. Solid-propellant combustion response function from direct measurement methods: ONERA experience. J. Propul. Power 15(6):837-43.

3. Cauty, F. 2006. Investigation in energetic materials combustion: A strategy for numerical simulation validation. AIAA Paper No. 2006-4742.

4. Cauty, F. 2007. Investigation in energetic materials combustion: solid propellant flame structure and temperature profile. AIAA Paper No. 2007-5863.

5. Desse, J.-M. 2002. Visualisations par techniques optiques: Ombroscopie, strioscopie, interférométrie différentielle et absolue. Revue Sciences et Techniques de la Défense 56.

6. Desse, J.-M., J. P. Bourez, F. Cauty, and C. Eradès. 2005. Développement des techniques de visualisation pour l'étude des matériaux énergétiques. Colloque FLUVISU11, ECL Ecully. France.

7. Weinstein, L. 1991. An improved large-field focusing Schlieren system. AIAA Paper No. 1991-0567.

8. Weinstein, L. 1993. Large-field high-brightness focusing Schlieren system. AIAA J. 31(7):1250-55.

9. Cauty, F., C. Eradès, and J.-M. Desse. 2009. Strioscopie focalisée appliquée à la combustion des propergols solides. 13ème Congrès Français de Visualisation et de Traitement d'Images en Mécanique des Fluides. ISBN 978-2-918241-01-0. Reims, France.

10. National Institute of Health. ImageJ. http://rsbweb.nih.gov/ij/index.html. 